\title{
Simultaneous Image Reconstruction and Feature Learning with 3D-CNNs for Image Set-Based Classification
}

\author{
XINYU ZHANG, Wuhan University \\ XIAOCUI LI, Huazhong University of Science and Technology \\ XIAO-YUAN JING, Guangdong University of Petrochemical Technology \\ LI CHENG, Wuhan University
}

\begin{abstract}
Image set-based classification has attracted substantial research interest because of its broad applications. Recently, lots of methods based on feature learning or dictionary learning have been developed to solve this problem, and some of them have made gratifying achievements. However, most of them transform the image set into a 2D matrix or use 2D convolutional neural networks (CNNs) for feature learning, so the spatial and temporal information is missing. At the same time, these methods extract features from original images in which there may exist huge intra-class diversity. To explore a possible solution to these issues, we propose a simultaneous image reconstruction with deep learning and feature learning with 3D-CNNs (SIRFL) for image set classification. The proposed SIRFL approach consists of a deep image reconstruction network and a 3DCNN-based feature learning network. The deep image reconstruction network is used to reduce the diversity of images from the same set, and the feature learning network can effectively retain spatial and temporal information by using 3D-CNNs. Extensive experimental results on five widely used datasets show that our SIRFL approach is a strong competitor for the state-of-the-art image set classification methods.
\end{abstract}

CCS Concepts: • Computing methodologies $\rightarrow$ Artificial intelligence; Computer vision;

Additional Key Words and Phrases: Image set-based classification, feature learning, deep reconstruction, 3D-CNNs

\section{ACM Reference format:}

Xinyu Zhang, Xiaocui Li, Xiao-Yuan Jing, and Li Cheng. 2021. Simultaneous Image Reconstruction and Feature Learning with 3D-CNNs for Image Set-Based Classification. ACM/IMS Trans. Data Sci. 2, 2, Article 12 (March 2021), 13 pages.

https://doi.org/10.1145/3420037

X. Zhang and X. Li are co-first authors of this article.

This work was supported by the NSFC-Key Project under grant 61933013, the NSFC-Key Project of General Technology Fundamental Research United Fund under grant U1736211, the Key Project of Natural Science Foundation of Hubei Province under grant 2018CFA024, the Natural Science Foundation of Guangdong Province under grant 2019A1515011076, the Innovation Group of Guangdong Education Department under grant 2018KCXTD019, and the Scientific Research Projects of Hunan Education Department under grant 14C0304.

Authors' addresses: X. Zhang and L. Cheng, School of Computer Science, Wuhan University, Wuhan, China; emails: zhangxinyu@whu.edu.cn, cjackl@126.com; X. Li, Wuhan National Laboratory for Optoelectronics and School of Computer Science and Technology, Huazhong University of Science and Technology, Wuhan, China; email: lxc@hust.edu.cn; X.-Y. Jing (corresponding author), School of Computer Science, Guangdong University of Petrochemical Technology, Maoming, China and School of Computer Science, Wuhan University, China; email: jingxy_2000@126.com.

Permission to make digital or hard copies of all or part of this work for personal or classroom use is granted without fee provided that copies are not made or distributed for profit or commercial advantage and that copies bear this notice and the full citation on the first page. Copyrights for components of this work owned by others than ACM must be honored. Abstracting with credit is permitted. To copy otherwise, or republish, to post on servers or to redistribute to lists, requires prior specific permission and/or a fee. Request permissions from permissions@acm.org.

(C) 2021 Association for Computing Machinery.

2577-3224/2021/03-ART12 \$15.00

https://doi.org/10.1145/3420037 


\section{INTRODUCTION}

With significant advances in camera technology and devices, large amounts of information is recorded in the form of image sets. However, image set-based classification can be widely used in various areas, including multi-view visual recognition [27, 37, 39], video-based surveillance $[4,38]$, and dynamic scene recognition, [29], among others. Therefore, more and more researchers pay attention to the problem of image set-based classification. Compared with single-image-based classification, image set-based classification is more appealing because the image set can provide much richer information with multiple samples. In fact, an image set can convey rich within-class variations of an object, which is helpful for classification. At the same time, it also will raise great challenges caused by changing illumination conditions, different backgrounds, viewpoint, occlusions, and disguise.

In past decades, many good methods have been addressed to solve the image set classification problem. Existing previous works were mostly developed under specific assumptions on distributions or geometrical structures, and there are two major steps involved in these methods. The first step is to find a suitable feature representation of an image set, and the second step is to define an appropriate distance to compute the similarity between features learned from the first step. At first, a Gaussian mixture model [1] is presented that learns highly non-linear but intrinsically lowdimensional manifolds. To facilitate modeling the image sets, Cevikalp and Triggs [2] designed a general geometric affine hull model to represent an image set. Then, some more efficient and accurate models are presented, such as presented elsewhere in the literature [11, 25, 34]. These methods use subspace learning or manifold learning to model image sets. In the work of Chen [3], a dual linear regression classification (DLRC) method is proposed. DLRC pays attention to minimizing the distance between the query set and the related train set. To improve the performance of DLRC, Feng et al. [7] introduced a pairwise linear regression classification (PLRC). However, the performance of these methods may degrade because of the problems of intra-class diversity and inter-class similarity.

Recently, the deep learning technique has achieved great success in many fields $[15,22]$ due to its highly learning capacity, and some deep learning-based methods $[6,12,16,25]$ have been developed to tackle the image set classification problem and obtain state-of-the-art classification performance. This family of methods usually extracts nonlinear transformation features by deep networks for each image set or class and then uses the learned features to measure the similarity of image sets. However, how to effectively represent the multiple instances for each image set and how to accurately compute the distances between different image sets remains an open problem and needs to be studied further.

\subsection{Motivation}

Many metric learning methods, including traditional metric learning and deep metric learning, have been addressed to solve the image set classification problem. Although reasonably good classification performance can be obtained, there remains much room for improvement. Specifically, most of the previous traditional metric learning works transform an image set into a $2 \mathrm{D}$ matrix for subsequent processing, which not only cannot learn enough discriminative features but also breaks the inherent spatial structure of the image set. Wu et al. [36] used the third-order tensor to represent the image set, which can effectively retain the spatial structure information. However, they separately represent the image set from three dimensions using a third-order tensor rather than thinking of the image set as an integrated whole. For existing deep metric learning methods solving the image set classification problem, they use 2D convolutional neural networks (CNNs) for feature learning, so the information between images is missing. Moreover, the preceding 


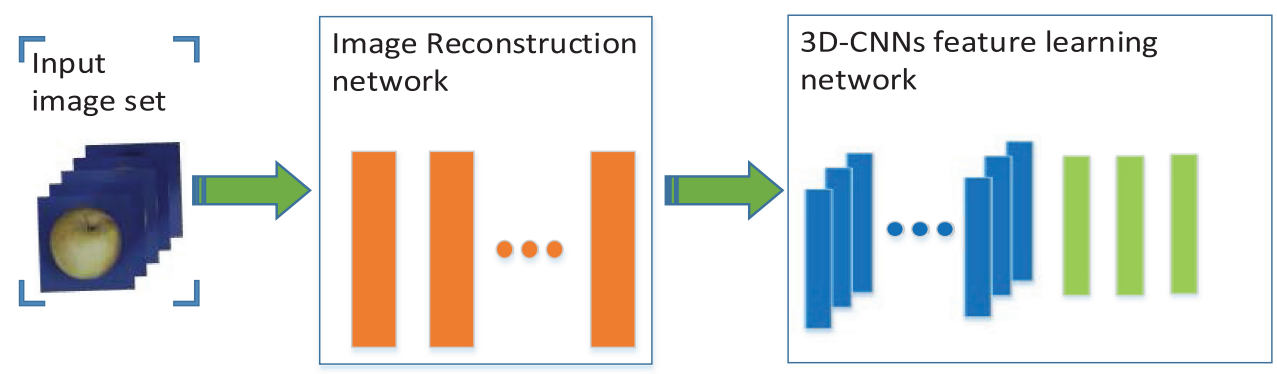

Fig. 1. The basic idea of our proposed SIRFL approach to image set classification. SIRFL contains two subnetworks: an image reconstruction network and a 3D-CNN feature learning network. Details of the two subnetworks are shown in following section.

methods learn the metric model from an original raw image, which may contain some noisy and irrelevant components.

Motivated by the preceding analysis, we designed SIRFL, a novel approach, by simultaneously using image reconstruction and feature learning with 3D-CNNs for image set-based classification. As shown later in Figure 1, the proposed approach consists of two subnetworks: a deep image reconstruction network and a $3 \mathrm{D}-\mathrm{CNN}$ feature learning network.

\subsection{Contributions}

The major contributions of our work can be summarized as follows:

(1) A joint deep metric learning framework is proposed for image set classification, which includes a deep image reconstruction subnetwork and a 3D-CNN-based feature learning subnetwork.

(2) To obtain more discriminate and robust features, we design a 3D-CNN-based feature learning network that can effectively retain the spatial and temporal information of an image set. At the same time, to facilitate feature extracting and learning, we design a deep image reconstruction network to reduce the diversity between images.

(3) We have conducted several experiments on five publicly available image set datasets to demonstrate the effectiveness of our proposed approach. The experiment results show that our approach is a strong competitor for state-of-the-art image set classification methods.

\section{RELATED WORK}

\subsection{Image Reconstruction-Based Methods}

Image reconstruction-based methods try to reconstruct the original image to obtain a lowerdimensional but more representational feature image. In the work of Hayat et al. [12], DRM-WV, a deep reconstruction model with weighted voting method is proposed to learn class-specific deep reconstruction models under the learned class-specific models to minimize reconstruction errors. In the work of Shah et al. [28], a linear regression reconstruction model is presented, and in the work of Li et al. [21], the authors first utilized two sparse bases to reconstruct an image and then combined the adaptive sampling technique for different image blocks. However, these methods have high computational complexity.

Compared with these methods, the main differences are as follows. First, unlike the methods of Hayat et al. [12] and Shah et al. [28], which learn different reconstruction models for each class image set, our approach only uses a common reconstruction network for all image sets. Second, different from Hayat et al. [12], Shah et al. [28], and Li et al. [21], our model not only minimizes 
the reconstruction error between the reconstructed image and the original image but also requires that images from the same image set should be compact to each other.

\subsection{Image Set-Based Classification Methods}

In this section, we introduce the related work about image set-based classification methods. Generally speaking, existing mainstream image set classification methods can be roughly categorized into three types: (i) methods based on traditional space and metric learning, (ii) those based on dictionary learning, and (iii) those based on deep learning.

Traditional subspace and metric learning. Subspace learning-based methods aims to learn an embedding common space [9, 23]. Manifold discriminant analysis (MDA) [31] is a typical representative of subspace learning methods. The discriminant analysis on the Riemannian manifold of Gaussian distributions [33] method represents the image set as a Gaussian mixture model comprising several Gaussian components and seeks to discriminate Gaussian components from different classes. Gao et al. [9] proposed a multi-model fusion metric learning method in which an image set is jointly represented by the mean vector, subspace, and covariance matrix. Metric learning-based methods measure the distance of image sets by feature transforming or feature learning. However, most of these methods cannot deal with nonlinear problems effectively.

Dictionary learning. Dictionary learning aims to seek a set of atoms for sparse representation of the input samples [14], where each sample is linearly represented by a small number of atoms. Chen et al. [5] proposed a dictionary learning approach by building one dictionary for each image set and measured the similarity of image sets with these learned dictionaries. To obtain more good classification performance, a discriminant tensor dictionary learning with neighbor uncorrelation (DTDLNU) method is proposed [36]. Gao et al. [8] designed a new image set classification framework, kernel pairwise linear regression classification (KPLRC). KPLRC embeds the related and unrelated gallery sets and probe sets into the high-dimensional Hilbert space, so the data become more linear separable. However, these approaches are a generative model and the dictionaries are learned from the original raw image, which may contain some noisy and irrelevant components.

Deep learning. Generally speaking, this type of method aims to seek high-level representational features by learning hierarchical features from raw data. Hayat et al. [10] first used the deep learning model to solve the image set classification problem. Lu et al. [25] combined the deep learning technique with manifold learning. Cheng et al. [6] proposed a duplex deep metric learning model with different objectives used for feature learning and image set classification. However, there are two shortcomings in these methods. First, the testing computational time is high. To determine the label of the test image set, they should first compute the distance of each image pair, one from the test image set and one from the training sets, then employ a voting strategy or compute average distance. Second, they use 2D-CNNs for feature learning, so the spatial and temporal information contained in the image set is not effectively extracted.

\section{PROPOSED METHOD}

The basic idea of our proposed SIRFL approach is shown in Figure 1, and the following sections will present details of the proposed approach.

\subsection{Deep Image Reconstruction Model}

We first define a deep image reconstruction model (DIRM). The architecture of our DIRM is summarized in Figure 2. As shown in the figure, our DIRM has two parts: an encoder subnet and a 


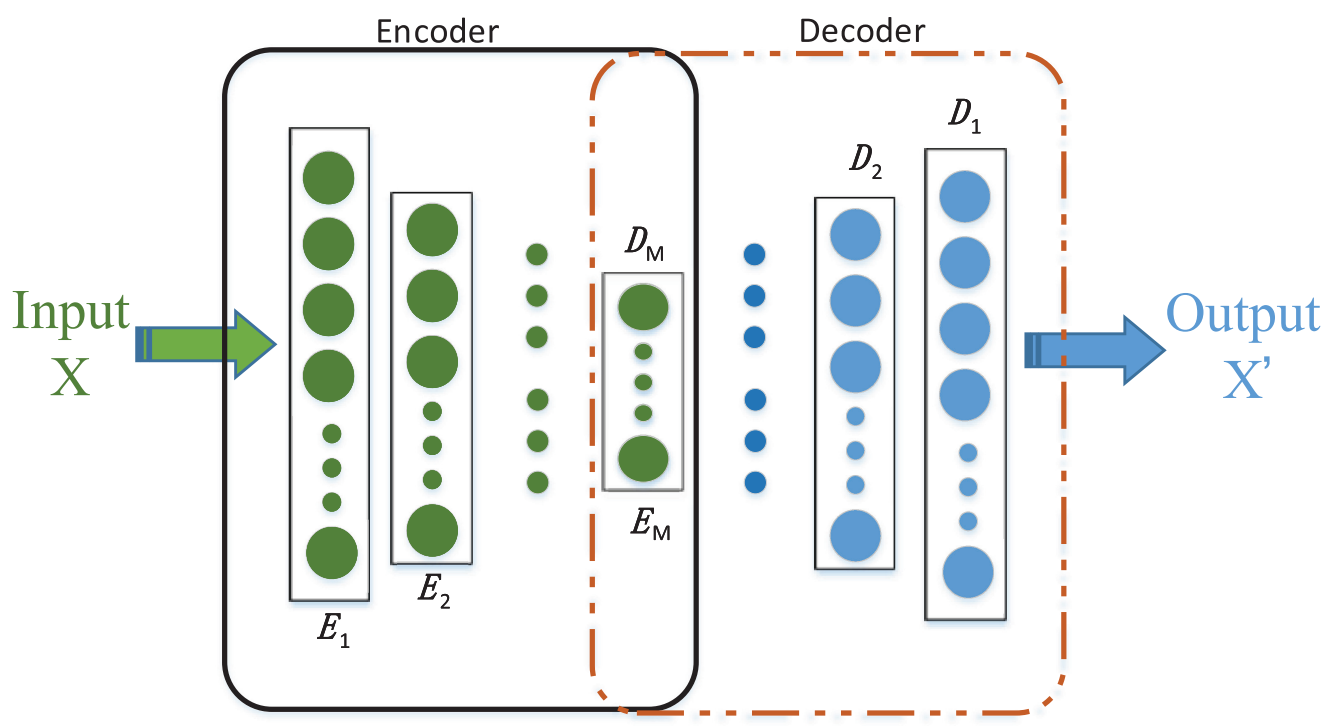

Fig. 2. The structure of DIRM. DIRM includes two parts: an encoder subnet and a symmetric decoder subnet, and each subnet contains $M$ layers.

symmetric decoder subnet. Both of them have $M$ layers, with an $M$ four layer. The encoder part finds a lower-dimensional but more meaningful representation of the raw image, and the decoder part reconstructs the input raw image by deconvolution.

For an image $x_{i} \in \mathbb{R}^{d}$ from the image set $X=x_{1}, x_{2}, \ldots, x_{N} \in \mathbb{R}^{d \times N}$, where $N$ is the number of images, let $E_{m}\left(x_{i}\right)$ be the output of the $m$ layer of the encoder subnet, where $m=1,2, \ldots, M$, and let $D_{m}\left(x_{i}\right)$ be the input of the $m$ layer of the decoder subnet, where $m=M, \ldots, 2,1$, and there are $k_{m}$ neurons in the $m$ encoder and decoder subnet. Then, $E_{m}\left(x_{i}\right)$ and $D_{m}\left(x_{i}\right)$ can be computed as

$$
\begin{gathered}
E_{m}\left(x_{i}\right)=\varphi\left(W_{m}^{e} E_{m-1}\left(x_{i}\right)+b_{m}^{e}\right), \\
D_{m}\left(x_{i}\right)=\varphi\left(W_{m}^{d} D_{m+1}\left(x_{i}\right)+b_{m}^{d}\right),
\end{gathered}
$$

where $W_{m}^{e} \in \mathbb{R}^{k_{m} \times k_{m-1}}$ and $b_{m}^{e} \in \mathbb{R}^{k_{m}}$ are the weight matrix and bias of the parameters in the $m^{t h}$ layer of the encoder subnet, $W_{m}^{d} \in \mathbb{R}^{k_{m} \times k_{m+1}}$ and $b_{m}^{d} \in \mathbb{R}^{k_{m}}$ are the weight matrix and bias of the parameters in the $m^{\text {th }}$ layer of the decoder subnet, and $\varphi(\cdot)$ is a nonlinear activation function that operates component wisely, such as widely used sigmoid or tanh functions. For the first layer of the dncoder, $E_{1}\left(x_{i}\right)=x_{i}, k_{1}=d$.

Different from the method of Hayat et al. [12], which only requires that the model can minimize the reconstruction error between the reconstructed image and input image, our DIRM also requires that the reconstructed images from the same image set should be compact to each other. According to this point, we design the objective function of our DIRM as follows:

$$
\begin{aligned}
& \min J\left(W_{m}^{e}, b_{m}^{e}, W_{m}^{d}, b_{m}^{d}\right) \\
& =\left(J_{1}\left(E_{m}, D_{m}\right)+\lambda_{1} J_{2}\left(E_{m}\right)+\frac{\lambda_{2}}{2}\left(\left\|W_{m}^{e}\right\|_{F}^{2}+\left\|W_{m}^{d}\right\|_{F}^{2}\right)\right),
\end{aligned}
$$


Table 1. Details of 3D-CNN Structure

\begin{tabular}{lcccc}
\hline Layer & Input Size & Output Size & Kernel & Stride \\
\hline Conv1 & $16 \times 20 \times 20 \times 1$ & $12 \times 16 \times 16 \times 16$ & $5 \times 5 \times 5$ & 1 \\
Pool2 & $12 \times 16 \times 16 \times 16$ & $12 \times 8 \times 8 \times 16$ & $1 \times 2 \times 2$ & $1 \times 2 \times 2$ \\
Conv3 & $12 \times 8 \times 8 \times 16$ & $10 \times 6 \times 6 \times 32$ & $3 \times 3 \times 3$ & 1 \\
Pool4 & $10 \times 6 \times 6 \times 32$ & $5 \times 3 \times 3 \times 32$ & $2 \times 2 \times 2$ & $2 \times 2 \times 2$ \\
Conv5 & $5 \times 3 \times 3 \times 32$ & $4 \times 2 \times 2 \times 64$ & $2 \times 2 \times 2$ & 1 \\
FC6 & $4 \times 2 \times 2 \times 64$ & 128 & - & - \\
FC7 & 128 & 64 & - & - \\
\hline
\end{tabular}

where $\lambda_{1}$ and $\lambda_{2}$ are tunable positive regularization parameters that control the relative importance of these three terms. $J_{1}$ is the reconstruction error, which is computed by

$$
\begin{aligned}
& J_{1}\left(E_{m}, D_{m}\right)=\frac{1}{2}\left(\left\|E_{m}\left(x_{i}\right)-D_{m}\left(x_{i}\right)\right\|_{2}^{2}\right. \\
& =\frac{1}{2}\left\|\varphi\left(W_{m}^{e} E_{m-1}\left(x_{i}\right)+b_{m}^{e}\right)-\varphi\left(W_{m}^{d} D_{m+1}\left(x_{i}\right)+b_{m}^{d}\right)\right\|_{2}^{2} .
\end{aligned}
$$

$J_{2}$ is a metric learning regularization term, which enforce the features to have small intra-set scatter. $J_{2}$ is defined as follows:

$$
\begin{aligned}
& J_{2}\left(E_{m}\right)=\frac{1}{2}\left(\left\|E_{m}\left(x_{i}\right)-E_{m}\left(u_{x}\right)\right\|_{2}^{2}\right. \\
& =\frac{1}{2}\left\|\varphi\left(W_{m}^{e} E_{m-1}\left(x_{i}\right)+b_{m}^{e}\right)-\varphi\left(W_{m}^{e} E_{m-1}\left(u_{x}\right)+b_{m}^{e}\right)\right\|_{2}^{2},
\end{aligned}
$$

where $u_{x}$ is the mean of image set $X$.

The third term in Equation (3) is a weight decay term to help preventing overfitting.

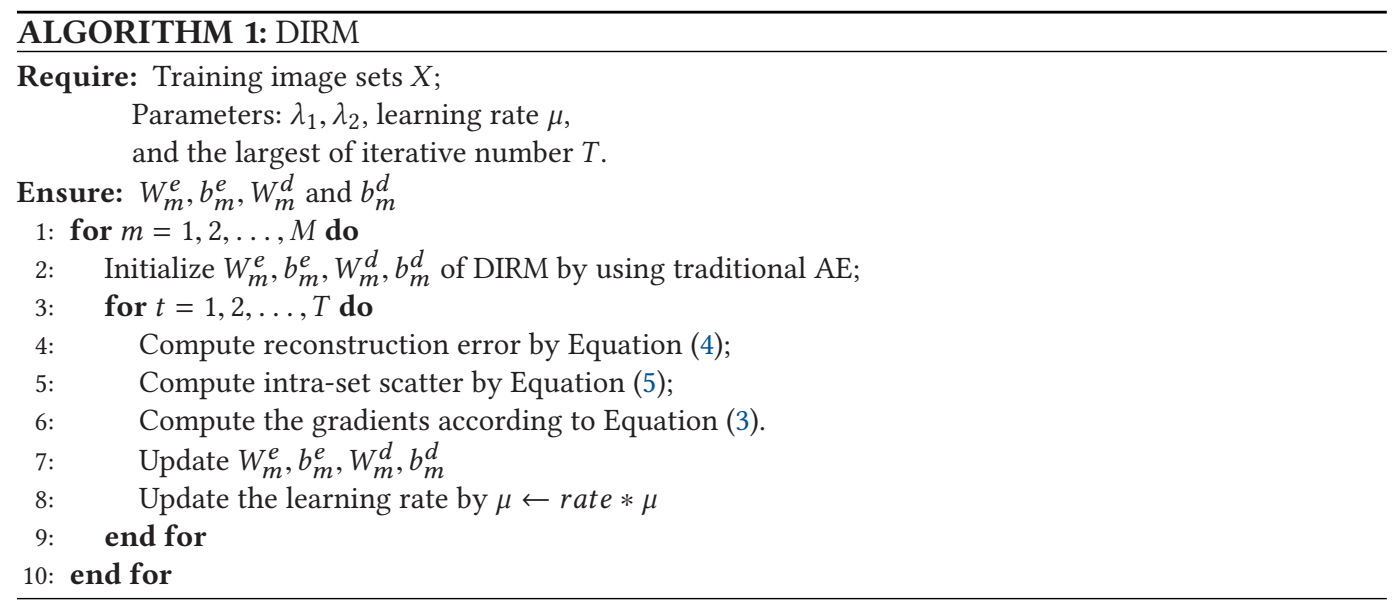

To solve the optimization problem in Equation (3), we use the stochastic sub-gradient descent method to update the parameters $W_{m}^{e}, b_{m}^{e}, W_{m}^{d}$, and $b_{m}^{d}$.

\subsection{D-CNN-Based Feature Learning}

For a reconstructed image set $X_{i}$ by DIRM, we extract the features of $X_{i}$ by passing it into a 3D$\mathrm{CNN}$ for metric learning. The structure of 3D-CNNs is shown in Figure 3, and Table 1 explains the details of network structure. 


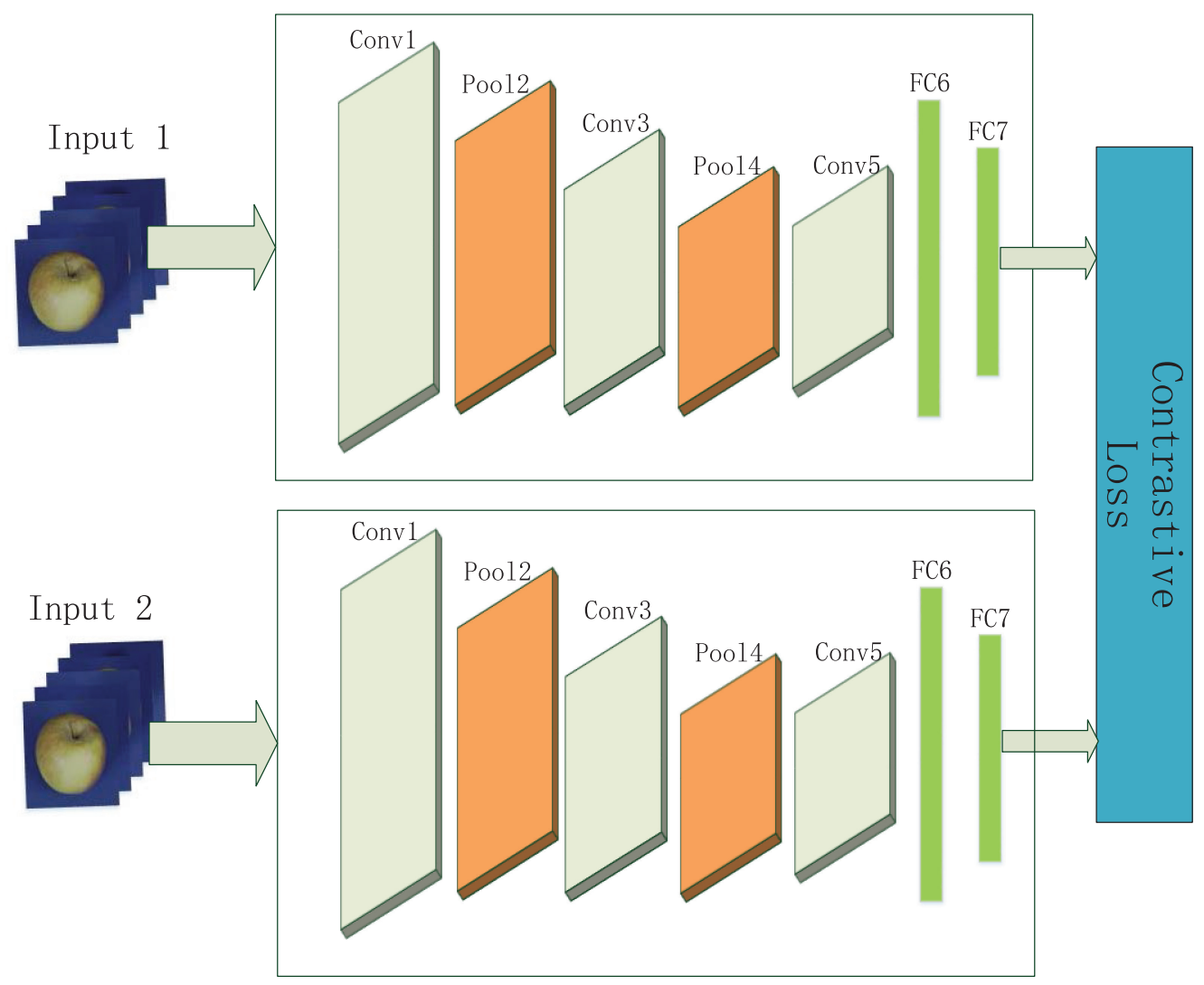

Fig. 3. Structure of 3D-CNN-based feature learning.

To measure the performance of the features learned from the network, we use contrastive loss as the discriminative distance metric to optimize the learning process. The contrastive loss function in our proposed approach is defined as follows:

$$
L_{W}(Y, X)=\frac{1}{N} \sum_{i=1}^{N} L_{W}\left(Y_{i},\left(X_{1}, X_{2}\right)_{i}\right)+\lambda\|W\|_{F}^{2},
$$

where $N$ is the number of training pairs, $\left(X_{1}, X_{2}\right)_{i}$ is the $i^{t h}$ input pair, $Y_{i} \in\{0,1\}$ (1 if the image set pair is from the same object, and 0 otherwise) is the corresponding label of the input pair, $\lambda\|W\|_{F}^{2}$ is a regular term to prevent overfitting ( $\lambda$ is a regularization parameter), and $L_{W}\left(Y_{i},\left(X_{1}, X_{2}\right)_{i}\right)$ is defined as follows:

$$
\begin{aligned}
& L_{W}\left(Y_{i},\left(X_{1}, X_{2}\right)_{i}\right)=Y_{i} D_{W}\left(X_{1}, X_{2}\right) \\
& +\left(1-Y_{i}\right) \max \left(\mu-D_{W}\left(X_{1}, X_{2}\right), 0\right),
\end{aligned}
$$

where $\mu$ is a predefined margin enforced on negative pairs and $D_{W}\left(X_{1}, X_{2}\right)$ is the Euclidean distance between the outputs of the 3D-CNN-based feature learning network with $\left(X_{1}, X_{2}\right)$ as the input. 


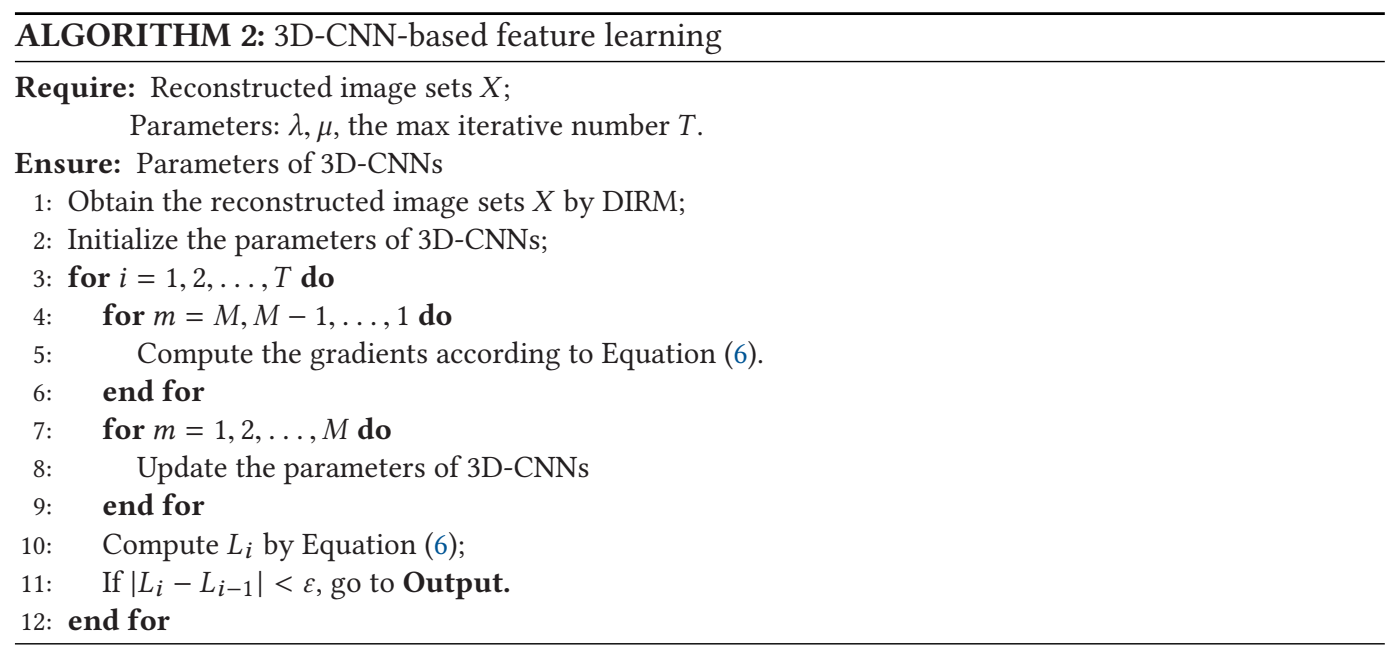

\subsection{The Classification Scheme of SIRFL}

Given a testing image set $X_{\text {test }}$, we compute the distance between the testing image set $X_{\text {test }}$ and each training image set $X_{\text {train }}^{i}$, and assign a label $L_{\text {test }}$ to the testing image set $X_{\text {test }}$ as follows:

$$
L_{\text {text }}=\arg \min _{i} D_{W}\left(X_{\text {test }}, X_{\text {train }}^{i}\right),
$$

where $D_{W}\left(X_{\text {test }}, X_{\text {train }}^{i}\right)$ is the Euclidean distance between the outputs of the 3D-CNN-based feature learning with $\left(X_{\text {test }}, X_{\text {train }}^{i}\right)$ as the input. The label of $X_{\text {train }}^{i}$ is the classification label of $L_{\text {test }}$.

\section{EXPERIMENTAL RESULTS}

\subsection{Compared Methods}

In the experiments, we compare our SIRFL with four categories of state-of-the-art-related methods including:

(1) Image reconstruction: DRM-WV [12] and LRC [28].

(2) Traditional subspace and metric learning: MDA [31], CDL [32], and SANP [13].

(3) Dictionary learning: DTDLNU [36] and SFDL [24].

(4) Deep learning: DML [6], DMK [30], D-SFDL [26], and MMDML [25].

\subsection{Datasets}

The performance of our proposed approach is evaluated on five publicly available datasets including Honda/UCSD [19], YouTube Celebrities (YTC) [17], YouTube Faces (YTF) [36], and PubFig face datasets [18], and the ETH-80 object dataset [20]. Honda contains 59 face video sequences from 20 different persons. YTC contains 1,910 video sequences of 47 celebrities from YouTube. YTF contains 3,425 videos of 1,596 subjects downloaded from YouTube. PubFig contains 58,797 images of 200 different persons. ETH-80 is a dataset involving eight different categories visual object.

\subsection{Experimental Settings}

For the Honda, YTC, and YTF datasets, we employ the face detector algorithm to detect faces in each image and resize the detected face images to $20 \times 20$. For the PubFig dataset, we crop the face region according to the provided bounding box and also resize the cropped faces to $20 \times 20$. For 
Table 2. Experimental Results of SIRFL and Compared Methods on Five Datasets

\begin{tabular}{lccccc}
\hline \multicolumn{1}{c}{ Method } & Honda & YTC & YTF & PubFig & ETH-80 \\
\hline DRW-MV & $\mathbf{1 0 0 . 0}$ & 71.3 & 46.9 & 88.6 & 98.1 \\
LRC & $\mathbf{1 0 0 . 0}$ & 66.4 & 70.6 & 73.1 & 94.8 \\
MDA & 94.5 & 68.1 & 66.3 & 48.6 & 89.2 \\
CDL & 97.4 & 69.7 & 72.1 & 65.5 & 91.5 \\
SANP & 95.3 & 68.4 & 76.7 & 78.5 & 80.5 \\
DTDLNU & 98.7 & 80.2 & 85.2 & 83.4 & 94.2 \\
SFDL & 98.5 & 76.9 & 80.2 & 78.5 & 90.5 \\
DML & $\mathbf{1 0 0 . 0}$ & 82.1 & 82.3 & 84.7 & $\mathbf{1 0 0 . 0}$ \\
DMK & 97.4 & 80.3 & 78.5 & 82.8 & 96.8 \\
D-SFDL & $\mathbf{1 0 0 . 0}$ & 79.5 & 77.6 & 83.5 & 92.3 \\
MMDML & $\mathbf{1 0 0 . 0}$ & 78.5 & 81.9 & 82.5 & 94.5 \\
SIRFL (ours) & $\mathbf{1 0 0 . 0}$ & $\mathbf{8 5 . 4}$ & $\mathbf{8 7 . 6}$ & $\mathbf{9 0 . 1}$ & 97.6 \\
\hline
\end{tabular}

The best result is in bold face.

the ETH- 80 dataset, all images are segmented from the simple background and scale to $20 \times 20$ for image set classification.

To make a fair comparison with related image set classification methods, we followed the protocol used in other works $[25,33,36]$. For the Honda dataset, we conducted experiments 10 times by randomly selecting different training and testing image sets. For the YTC dataset, we employed the 10 -fold cross-validation strategy. In each fold, three video sequences of each person are randomly selected for training, and the remaining six are used for testing. For the YTF dataset, we followed the standard evaluation protocol [35]. For the PubFig dataset, we used one fold for training and the remaining two for testing by randomly selecting different folds for training and testing. For the ETH-80 dataset, we randomly selected half of 10 image sets from each object for training and the remaining for testing. In addition, one video was regarded as an image set.

For our proposed approach SIRFL, it contains a deep image reconstruction network (DIRM) and a 3D-CNN-based feature learning network. DIRM includes an encoder subnet and a symmetric decoder subnet. We set $M$ to 4 , and the layers are set as 400,300, 200, 100, 200, 300, and 400, respectively. The parameters $\lambda_{1}$ and $\lambda_{2}$ are empirically set as 0.1 and 0.01 . We use the traditional AE strategy to initialize the weights of our DIRM. For the 3D-CNN-based feature learning network, structure details are shown in Table 1. For 3D-CNNs, if a video has more than 16 frames, we randomly select 16 consecutive frames to extract the features.

\subsection{Results and Analysis}

Comparison with the state of the art. Table 2 reports average classification rates of our proposed approach and 11 compared methods on all five datasets. From the results of Table 2, we can clearly see that our SIRFL approach can achieve better performance than most of the compared methods on all five datasets.

Specifically, compared with traditional methods, including subspace learning, metric learning, and dictionary learning, our SIRFL combines deep learning to learn more powerful and discriminative features. Compared with existing deep learning-based methods, our SIRFL also achieves better performance. The reasons are twofold. First, we design our DIRM to reduce the diversity of between images from the same image set. Second, we employ 3D-CNNs for feature learning, which can extract spatial and temporal information so that more discriminative features are obtained. 
Table 3. Experimental Results of SIRFL and Three Modified Version of SIRFL on Five Datasets

\begin{tabular}{lcccc}
\hline Dataset & SIRFL $_{p 1}$ & SIRFL $_{p 2}$ & SIRFL $_{2 D}$ & SIRFL \\
\hline Honda & 94.4 & 96.7 & 98.6 & $\mathbf{1 0 0 . 0}$ \\
YTC & 76.5 & 81.4 & 84.2 & $\mathbf{8 5 . 4}$ \\
YTF & 73.4 & 78.9 & 83.1 & $\mathbf{8 7 . 6}$ \\
PubFig & 80.2 & 81.3 & 86.8 & $\mathbf{9 0 . 1}$ \\
ETH-80 & 92.5 & 93.2 & 96.8 & $\mathbf{9 7 . 6}$ \\
\hline
\end{tabular}

The best result is in bold face.

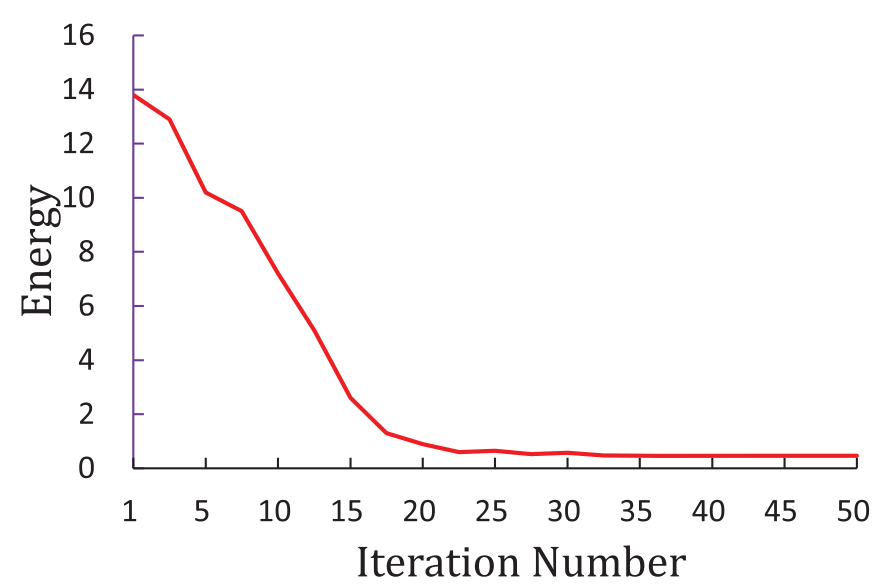

Fig. 4. Convergence curve of our approach on the YTC dataset.

Evaluation of DIRM and 3D-CNN -based feature learning. To evaluate the effect of DIRM and 3DCNN-based feature learning, we generate three modified versions of SIRFL and separately name them as "SIRFL $p 1$ " (just using DIRM), "SIRFL $p 2$ " (just using 3D-CNN-based feature learning), and "SIRFL $2 D$ " (using DIRM and 2D-CNN-based feature learning). The experimental results are shown in Table 3.

From the results of Table 3, we can observe that SIRFL achieves the best classification performance, which demonstrates that DIRM can reduce the diversity of images and 3D-CNN-based feature learning can extract spatial and temporal information so that more robust and discriminative features can be exploited to improve classification performance.

Convergence and computational time analysis. Last, we analyze the convergence of our SIRFL approach and the computational time of all competing methods. Our hardware configuration comprises a 3.6-GHz four-core CPU and 24 GB of RAM. Figure 4 and Figure 5 plot the value of the objective function of our approach versus iterations on the YTC and ETH-80 datasets respectively. From the result, we can clearly see that our proposed approach converges in about 30 iterations. Similar results can be seen in other datasets.

Table 4 shows the computational time of different methods on the YTC dataset. From the results in the table, we can see that our SIRFL approach requires more training time than that of traditional methods, but it is comparable to that of deep learning-based methods. In addition, the testing time of SIRFL is less than almost all compared methods except MDA. 


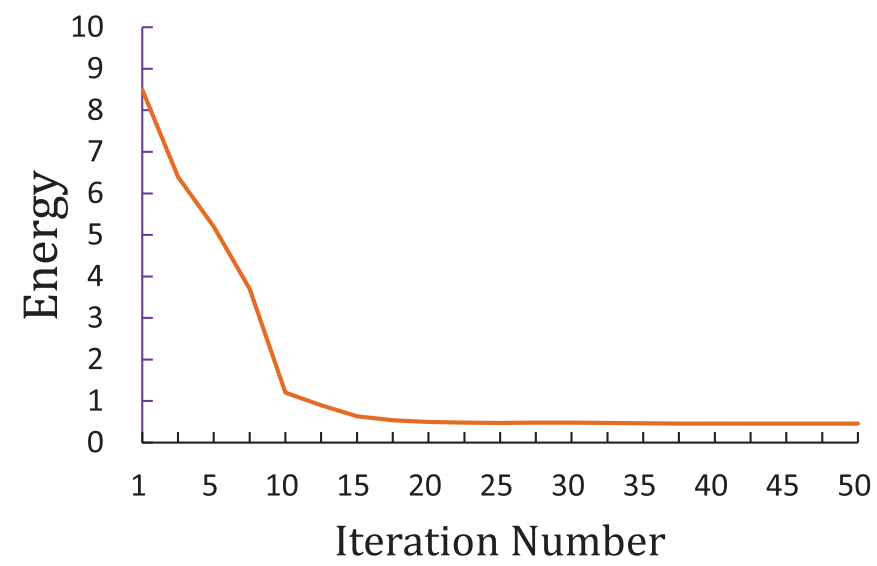

Fig. 5. Convergence curve of our approach on the ETH-80 dataset.

Table 4. Computation Time (Seconds) of SIRFL and Some Compared Methods on YTC for Training (Total) and Testing (Classification of One Image Set)

\begin{tabular}{lcc}
\hline Method & Training & Testing \\
\hline DRW-MV & 3873.4 & 4.8 \\
MDA & 183.9 & 3.3 \\
CDL & 68.3 & 12.4 \\
SANP & N.A & 47.2 \\
DTDLNU & 3605.9 & 157.1 \\
SFDL & 7518.2 & 6.5 \\
D-SFDL & 9632.8 & 7.8 \\
MMDML & 1534.3 & 5.4 \\
SIRFL & 6235.5 & 4.2 \\
\hline
\end{tabular}

\section{CONCLUSION AND FUTURE WORK}

In this article, we have proposed a novel image set classification approach by combining deep image reconstruction with 3D-CNN feature learning (SIRFL). SIRFL comprises a DIRM and a 3DCNN-based feature learning network. DIRM is mainly used to eliminate the diversity between images from one set, and the 3D-CNN-based feature learning network is designed for extracting spatial and temporal information. By doing so, the obtained features will have favorable discriminability. Experimental results on five popular datasets demonstrate that our SIRFL approach achieves better classification performance than the state-of-the-art methods.

In this work, our approach consists of two training steps, which is not an end-to-end method. Therefore, we will integrate these two steps to form an end-to-end model. In addition, we will further enhance the performance of our proposed approach and apply it to other compute vision applications, such as image set-based person re-identification, video-based surveillance, and dynamic scene recognition.

\section{ACKNOWLEDGMENTS}

The authors would like to thank the editor, the associate editor, and anonymous reviewers for their constructive comments in helping to improve our work. 


\section{REFERENCES}

[1] Ognjen Arandjelovic, Gregory Shakhnarovich, John Fisher, Roberto Cipolla, and Trevor Darrell. 2005. Face recognition with image sets using manifold density divergence. In Proceedings of CVPR. 581-588.

[2] Hakan Cevikalp and Bill Triggs. 2010. Face recognition based on image sets. In Proceedings of CVPR. 2567-2573.

[3] Liang Chen. 2014. Dual linear regression based classification for face cluster recognition. In Proceedings of CVPR. 2673-2680.

[4] Weihua Chen, Xiaotang Chen, Jianguo Zhang, and Kaiqi Huang. 2017. A multi-task deep network for person reidentification. In Proceedings of AAAI. 3988-3994.

[5] Yi Chen Chen, Vishal M. Patel, P. Jonathon Phillips, and Rama Chellappa. 2015. Dictionary-based face and person recognition from unconstrained video. IEEE Access 3 (2015), 1783-1798.

[6] Gong Cheng, Peicheng Zhou, and Junwei Han. 2018. Duplex metric learning for image set classification. IEEE Transactions on Image Processing 27, 1 (2018), 281-292.

[7] Qingxiang Feng, Yicong Zhou, and Rushi Lan. 2016. Pairwise linear regression classification for image set retrieval. In Proceedings of CVPR. 4865-4872.

[8] Xizhan Gao, Quansen Sun, Haitao Xu, and Jianqiang Gao. 2020. Sparse and collaborative representation based kernel pairwise linear regression for image set classification. Expert Systems with Applications 140 (2020), 112886.

[9] Xizhan Gao, Quansen Sun, Haitao Xu, Dong Wei, and Jianqiang Gao. 2019. Multi-model fusion metric learning for image set classification. Knowledge-Based Systems 164 (2019), 253-264.

[10] Munawar Hayat, Mohammed Bennamoun, and Senjian An. 2014. Learning non-linear reconstruction models for image set classification. In Proceedings of CVPR. 1915-1922.

[11] Munawar Hayat, Mohammed Bennamoun, and Senjian An. 2014. Reverse training: An efficient approach for image set classification. In Proceedings of ECCV. 784-799.

[12] Munawar Hayat, Mohammed Bennamoun, and Senjian An. 2015. Deep reconstruction models for image set classification. IEEE Transactions on Pattern Analysis and Machine Intelligence 37, 4 (2015), 713-727.

[13] Yiqun Hu, Ajmal S. Mian, and Robyn A. Owens. 2011. Sparse approximated nearest points for image set classification. In Proceedings of CVPR. 121-128.

[14] Peiguang Jing, Yuting Su, Zhengnan Li, Jing Liu, and Liqiang Nie. 2019. Low-rank regularized tensor discriminant representation for image set classification. Signal Processing 156 (2019), 62-70.

[15] Xiao-Yuan Jing, Xinyu Zhang, Xiaoke Zhu, Fei Wu, Xinge You, Yang Gao, Shiguang Shan, and Jingyu Yang. 2021. Multiset feature learning for highly imbalanced data classification. IEEE Transactions on Pattern Analysis and Machine Intelligence 43, 1 (2021), 139-156.

[16] Andrej Karpathy, George Toderici, Sanketh Shetty, Thomas Leung, Rahul Sukthankar, and Fei Fei Li. 2014. Large-scale video classification with convolutional neural networks. In Proceedings of CVPR. 1725-1732.

[17] Minyoung Kim, Sanjiv Kumar, Vladimir Pavlovic, and Henry A. Rowley. 2008. Face tracking and recognition with visual constraints in real-world videos. In Proceedings of CVPR. 1-8.

[18] Neeraj Kumar, Alexander C. Berg, Peter N. Belhumeur, and Shree K. Nayar. 2009. Attribute and simile classifiers for face verification. In Proceedings of ECCV. 365-372.

[19] Kuang-Chih Lee, Jeffrey Ho, Ming-Hsuan Yang, and David J. Kriegman. 2003. Video-based face recognition using probabilistic appearance manifolds. In Proceedings of CVPR. 313-320.

[20] Bastian Leibe and Bernt Schiele. 2003. Analyzing appearance and contour based methods for object categorization. In Proceedings of CVPR. 409-415.

[21] Huihui Li, Yan Zeng, and Ning Yang. 2018. Image reconstruction for compressed sensing based on joint sparse bases and adaptive sampling. Machine Vision and Applications 29, 1 (2018), 145-157.

[22] Xiaocui Li, Hongzhi Yin, Ke Zhou, and Xiaofang Zhou. 2020. Semi-supervised clustering with deep metric learning and graph embedding. World Wide Web 23, 2 (2020), 781-798.

[23] Bo Liu, Liping Jing, Jia Li, Jian Yu, Alex Gittens, and Michael W. Mahoney. 2019. Group collaborative representation for image set classification. International fournal of Computer Vision 127, 2 (2019), 181-206.

[24] Jiwen Lu, Gang Wang, Weihong Deng, and Pierre Moulin. 2014. Simultaneous feature and dictionary learning for image set based face recognition. In Proceedings of ECCV. 265-280.

[25] Jiwen Lu, Gang Wang, Weihong Deng, Pierre Moulin, and Jie Zhou. 2015. Multi-manifold deep metric learning for image set classification. In Proceedings of CVPR. 1137-1145.

[26] Jiwen Lu, Gang Wang, and Jie Zhou. 2017. Simultaneous feature and dictionary learning for image set based face recognition. IEEE Transactions on Image Processing 26, 8 (2017), 4042-4054.

[27] Liang Pang, Yanyan Lan, Jiafeng Guo, Jun Xu, Shengxian Wan, and Xueqi Cheng. 2016. Text matching as image recognition. In Proceedings of AAAI. 2793-2799. 
[28] Syed Afaq Ali Shah, Uzair Nadeem, Mohammed Bennamoun, Ferdous Ahmed Sohel, and Roberto Togneri. 2017. Efficient image set classification using linear regression based image reconstruction. In Proceedings of CVPR Workshops. 601-610.

[29] Xinhang Song, Luis Herranz, and Shuqiang Jiang. 2018. Depth CNNs for RGB-D scene recognition: Learning from scratch better than transferring from RGB-CNNs. arXiv:1801.06797

[30] Haoliang Sun, Xiantong Zhen, Yuanjie Zheng, Gongping Yang, Yilong Yin, and Shuo Li. 2017. Learning deep match kernels for image-set classification. In Proceedings of CVPR. 3307-3316.

[31] Ruiping Wang and Xilin Chen. 2009. Manifold discriminant analysis. In Proceedings of CVPR. 429-436.

[32] Ruiping Wang, Huimin Guo, Larry S. Davis, and Qionghai Dai. 2012. Covariance discriminative learning: A natural and efficient approach to image set classification. In Proceedings of CVPR. 2496-2503.

[33] Wen Wang, Ruiping Wang, Zhiwu Huang, Shiguang Shan, and Xilin Chen. 2018. Discriminant analysis on Riemannian manifold of Gaussian distributions for face recognition with image sets. IEEE Transactions on Image Processing 27, 1 (2018), 151-163.

[34] Wen Wang, Ruiping Wang, Shiguang Shan, and Xilin Chen. 2017. Prototype discriminative learning for image set classification. IEEE Signal Processing Letters 24, 9 (2017), 1318-1322.

[35] Lior Wolf, Tal Hassner, and Itay Maoz. 2011. Face recognition in unconstrained videos with matched background similarity. In Proceedings of CVPR. 529-534.

[36] Fei Wu, Xiao Yuan Jing, Wangmeng Zuo, Ruiping Wang, and Xiaoke Zhu. 2017. Discriminant tensor dictionary learning with neighbor uncorrelation for image set based classification. In Proceedings of IFCAI. 3069-3075.

[37] Man Zhang, Ran He, Dong Cao, Zhenan Sun, and Tieniu Tan. 2016. Simultaneous feature and sample reduction for image-set classification. In Proceedings of AAAI, Vol. 16. 1401-1407.

[38] Xiaoke Zhu, Xiao-Yuan Jing, Fei Wu, Yunhong Wang, Wangmeng Zuo, and Wei-Shi Zheng. 2017. Learning heterogeneous dictionary pair with feature projection matrix for pedestrian video retrieval via single query image. In Proceedings of AAAI. 4341-4348.

[39] Xiaoke Zhu, Xiao-Yuan Jing, Fei Wu, Di Wu, Li Cheng, Sen Li, and Ruimin Hu. 2017. Multi-kernel low-rank dictionary pair learning for multiple features based image classification. In Proceedings of AAAI. 2970-2976.

Received February 2020; revised July 2020; accepted August 2020 